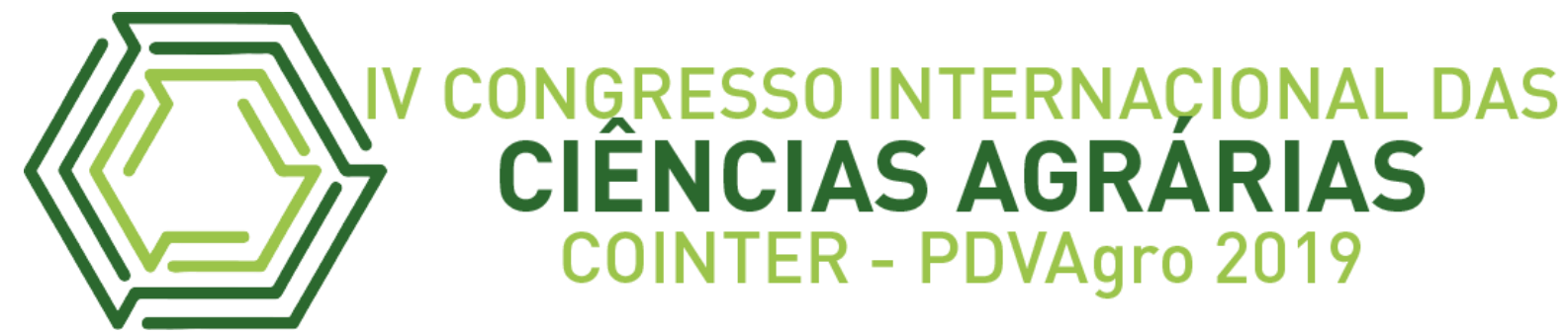

\title{
SIMULAÇÃO MATEMÁTICA DO COMPORTAMENTO HIDRÁULICO DE MICROASPERSORES AO LONGO DA LINHA LATERAL
}

\section{SIMULACIÓN MATEMÁTICA DEL COMPORTAMIENTO HIDRÁULICO DE MICROPERSORES DE LÍNEA LATERAL}

\section{MATH SIMULATION OF THE HYDRAULIC BEHAVIOR OF MICROPERSORS ALONG OF THE SIDE LINE}

\author{
Apresentação: Comunicação Oral
}

Julyane Silva Mendes Polycarpo ${ }^{1}$; Marco Antonio Silva²; Adriel Sales Coutinho ${ }^{3}$; Pollyanna Cavalcanti dos Santos de Souza ${ }^{4}$; João Audifax Cezar de Albuquerque Filho ${ }^{5}$

DOI: $\underline{\text { https://doi.org/10.31692/2526-7701.IVCOINTERPDVAgro.2019.0091 }}$

\begin{abstract}
Resumo
A água é um recurso natural imprescindível para a manutenção e desenvolvimento da humanidade. Atualmente há uma preocupação demasiada com a quantidade de água requerida para a irrigação, o qual pode ser justificada pelo decréscimo de sua disponibilidade tanto quantitativamente como qualitativamente, atenuando o interesse pela racionalização desse recurso. A prática da irrigação localizada comparada aos demais sistemas de irrigação, vem crescendo destacadamente no cenário mundial mostrando-se um mercado promissor, e tem particularmente na região semiárida uma maior aplicação devido ao seu uso proporcionar uniformidade e eficiência, além de minimizar os riscos de salinização. Objetivou-se com este estudo avaliar o comportamento hidráulico do gradiente de pressão e do comprimento ao longo da linha lateral de dois emissores tipo microaspersor, utilizando o modelo de $\mathrm{Wu}$ e Gitlin. O dimensionamento do projeto foi feito a partir de simulações matemáticas para dois microaspersores (bocal Rosa e Gelo). Para simulação do perfil de pressão ao longo da linha lateral, adotou o modelo de dimensionamento proposto por Wu e Gitlin, em que, foi calculada a descarga do emissor, a descarga total da linha lateral composta por 10 microaspersores, a viscosidade cinemática da água, a perda de carga, o fator de correção, a perda de carga no trecho e a perda de carga unitária. Sendo, então, obtido o diâmetro da linha lateral, o comprimento da linha lateral e a declividade. O microaspersor Rosa apresentou uma variação instável a laminar e do bocal Gelo um regime turbulento a totalmente turbulento. Conclui-se que o modelo aplicado foi eficaz no dimensionamento do gradiente de pressão ao longo da

\footnotetext{
${ }^{1}$ Graduanda em Gestão Ambiental, Instituto Federal de Pernambuco, julyanemendes@gmail.com

${ }^{2}$ Mestrando em Engenharia Agrícola, Universidade Federal Rural de Pernambuco, gillyart@hotmail.com

3 Mestrando em Engenharia Agrícola, Universidade Federal Rural de Pernambuco, adriel_sales2009@hotmail.com

${ }^{4}$ Graduanda em Bacharelado em Ciências Biológicas, Universidade Federal Rural de Pernambuco, pollycavalcanti.ss@hotmail.com

${ }^{5}$ Prof ${ }^{\circ}$ Doutor em Agronomia, Universidade Federal Rural de Pernambuco, jaudifaxfilho@yahoo.com.br
} 
linha lateral dos microaspersores de acordo com o comportamento hidráulico do sistema.

Palavras-Chave: Irrigação localizada, modelagem hidráulica, vazão-pressão.

\begin{abstract}
Resumen
El agua es un recurso natural indispensable para el mantenimiento y el desarrollo de la humanidad. Actualmente hay demasiada preocupación por la cantidad de agua requerida para el riego, lo que puede justificarse al disminuir su disponibilidad tanto cuantitativa como cualitativamente, atenuante el interés en racionalizar este recurso. La práctica del riego localizado en comparación con otros sistemas de riego, ha crecido de manera prominente en el escenario mundial mostrando un mercado prometedor, y tiene particularmente en la región semiárida una mayor aplicación debido a su uso para proporcionar uniformidad y eficiencia, además de minimizar los riesgos de salinización. El objetivo de este estudio fue evaluar el comportamiento hidráulico del gradiente de presión y la longitud a lo largo de la línea lateral de dos emisores de microaspersores, utilizando el modelo de $\mathrm{Wu}$ y Gitlin. El dimensionamiento del proyecto se realizó a partir de simulaciones matemáticas para dos microaspersores (boquilla Rosa y Hielo). Para la simulación del perfil de presión a lo largo de la línea lateral, adoptó el modelo de dimensionamiento propuesto por $\mathrm{Wu}$ y Gitlin, en el que, se calculó la descarga del emisor, la descarga total de la línea lateral compuesta por 10 microaspersores, la viscosidad cinemática del agua, la caída de presión, el factor de corrección, la caída de presión de estiramiento y la caída de presión de la unidad de carga. Siendo, entonces, obtenido el diámetro de la línea lateral, la longitud de la línea lateral y la pendiente. El microaspersor Rosa presentó una variación inestable a laminar y de la boquilla de Hielo un régimen turbulento a totalmente turbulento. Se concluyó que el modelo aplicado fue efectivo en el dimensionamiento del gradiente de presión a lo largo de la línea lateral de los microaspersores de acuerdo con el comportamiento hidráulico del sistema.
\end{abstract}

Palabras Clave: Riego localizado, modelado hidráulico, flujo de presión.

\begin{abstract}
The water is an indispensable natural resource for the maintenance and development of humanity. Currently there is preoccupation about the amount of water required for irrigation, which may be justified by decreasing its availability both quantitatively and qualitatively, mitigating the interest in rationalizing this resource. The practice of localized irrigation compared to the others irrigation systems, has been growing in the world scenario showing a promising market, and has in particular in the semi-arid region greater application due to its use provide uniformity and efficiency, besides minimizing the risks of salinization. The objective of this study was to evaluate the hydraulic behavior of the pressure gradient and length along the lateral line of two micro-sprinkler emitters, using the $\mathrm{Wu}$ and Gitlin model. The sizing of the project was made from mathematical simulations for two micro speakers (Rose and Ice nozzle). For simulation of the pressure profile along the lateral line, adopted the sizing model proposed by $\mathrm{Wu}$ and Gitlin, in which, the emitter discharge was calculated, the total discharge of the lateral line composed of 10 micro sprinklers, the kinematic viscosity of the water, the pressure drop, the correction factor, the pressure drop non-stretch and unit pressure drop. Then, the lateral line diameter, the lateral line length and the slope was
\end{abstract}


obtained. The Rose micro sprinkler presented an unstable variation to laminar and the nozzle Ice a turbulent to totally turbulent regime. It is concluded that the applied model was effective in dimensioning the pressure gradient along the lateral line to the microsprinklers according to the hydraulic behavior of the system

Keywords: Located irrigation, hydraulic modeling, pressure-flow.

\section{Introdução}

De acordo com Vörösmarty et al. (2010), a água é um recurso natural imprescindível para a manutenção e desenvolvimento da humanidade, onde se destaca seu uso no setor agrícola com um consumo equivalente a aproximadamente $75 \%$, valor este, muito acima de outras vertentes como o uso na indústria $20 \%$ e para utilização doméstica 5\% (D’ISEP, 2010).

Atualmente há uma preocupação demasiada com a quantidade de água requerida para a irrigação, a qual pode ser justificada pelo decréscimo de sua disponibilidade tanto quantitativamente como qualitativamente, atenuando o interesse pela racionalização desse recurso (WWDR, 2012, 2018; ANA, 2017). De acordo com os dados supracitados, o fato de a agricultura ser o maior responsável pelo consumo de água, indica a necessidade de uma renovação de ideias em torno da optimização e racionalização dos recursos hídricos (YU et al, 2010), tal investimento em tecnologias viáveis com a finalidade de reduzir a quantidade de aplicação de água via irrigação nas condições de baixa disponibilidade hídrica sem afetar a produção possibilitará no aumento da produção de alimentos para as próximas gerações (HERRERO et al.,2010).

Além da escassez generalizada dos recursos hídricos, em algumas regiões como o semiárido nordestino os efeitos da seca são ainda mais acentuados. O semiárido nordestino apresenta elevadas taxas de insolação, elevadas temperaturas e baixas amplitudes térmicas. Os totais pluviométricos são baixos e apresentam alta variabilidade no tempo e no espaço. Ocorrem, ainda, elevadas taxas de evapotranspiração e elevado déficit hídrico (ZANELLA, 2014).

Clark et al. (2007), caracteriza o sistema hidráulico de um sistema de irrigação a partir da composição das linhas principais, linhas de derivação, linhas lateral e dos emissores, os quais são responsáveis por disponibilizar a água para as plantas. $\mathrm{O}$ desempenho dos sistemas de irrigação depende muito do projeto hidráulico adequado, onde as principais tarefas do cálculo de dimensionamento hidráulico de um sistema de irrigação por microaspersores são 
determinar algumas características como (diâmetro e comprimento) das linhas laterais, das linhas de derivação e a linha principal, a pressão no início das linhas; a perda de carga e a vazão dos emissores, a fim de adequar o dimensionamento do sistema ainda em fase de projeto.

Wu (1997) destaca a necessidade de determinar a correta pressão no sistema para não o comprometer, pois altas pressões podem resultar em falhas na aplicação de água que tem como consequência afetar o crescimento e desenvolvimento da cultura (TAIZ et al., 2017), ou ainda resultar em lesões nas tubulações de irrigação (CLARK et al., 2007). Os autores Andrade e Ogliari (2013), descrevem a modelagem hidráulica como uma simplificação da realidade de sistemas complexos, o qual pode ser descrita por meio de equações. Por fim, conclui-se que o correto dimensionamento hidráulico de um sistema de irrigação garante a menor variação de vazão entre os emissores, com isso, garantindo uma aplicação uniforme da água.

A quantificação da pressão por sua vez é feita em decorrência da perda de carga e a equação de Blasius é do tipo potencial com o fator de atrito em função do número de Reynolds e de duas constantes adimensionais (SOUSA \& NETO, 2014). Pimenta et al. (2018), analisaram as principais equações explícitas utilizadas para a obtenção do fator atrito e elegeram a equação de Offor \& Alabi (2016a) como a mais eficiente.

Diante do exposto, objetivou-se com o presente trabalho propor uma equação explícita para o cálculo do fator de atrito de Darcy-weisbach e avaliar o comportamento hidráulico do gradiente de pressão e da vazão de um emissor tipo microaspersor ao longo da linha lateral.

\section{Fundamentação Teórica}

A irrigação é a prática agrícola que utiliza um conjunto de equipamentos e técnicas para suprir a deficiência total ou parcial de água para as plantas (MANTOVANI; BERNARDO; PALARETTI, 2009). Taiz et al.(2017), destaca a importância da água principalmente na agricultura, já que a deficiência hídrica compromete a produtividade das culturas, sua importância também está ligada ao transporte e absorção de nutrientes por meio de reações químicas na planta, o estresse hídrico, o excesso ou a carência de água afeta diretamente o crescimento e desenvolvimento da planta.

A prática da irrigação data desde as antigas civilizações, porém nos últimos anos seu desenvolvimento tem proporcionado o aumento da produção utilizando a mesma área ou até 
usando áreas menores, esta tecnologia possibilitou a China e a Índia de triplicar suas produções nos últimos 25 anos, por meio de investimentos na irrigação (FAO, 2011).

A agricultura irrigada se desenvolveu a princípio em regiões em que a quantidade, distribuição espacial e temporal das chuvas não eram capazes de suprir a demanda de água para as culturas (ANA, 2017).

De acordo com a Agência Nacional de Águas (ANA, 2017), a agricultura irrigada por retirar água do manancial para sua destinação (irrigação) classifica-se como uso consuntivo de água, ou seja, altera suas condições na medida em que a retira do ambiente e a maior parte é consumida pela evapotranspiração da planta e solo, não retornando diretamente aos corpos hídricos. Maupin et al. (2014) ressalta a semelhança dos dados de consumo de água para a irrigação com os dados dos EUA. A produção irrigada apresenta uma alta demanda por água, todavia, se tem como resposta o aumento da produção agrícola (FAO, 2011).

Assim como foi dito anteriormente, o desafio é desenvolver técnicas eficientes que tragam retorno social e econômico, porém deve-se haver o cuidado de não concorrer por água destinada ao abastecimento urbano (FGV, 2016). As comunidades rurais em países em desenvolvimento dependem fortemente da água, sua vulnerabilidade pode ser observada com referência nas seguintes médias: O setor absorve em média $84 \%$ dos impactos econômicos adversos das secas, e $25 \%$ de todos os danos causados por desastres relacionados ao clima (FAO, 2015).

Define-se o manejo de irrigação como um conjunto de métodos os quais possibilitarão estabelecer a quantidade de água necessária e o momento correto para realizar a irrigação (Mantovani et al., 2009; Dominguez et al., 2012; Frizzone et al., 2012). Conclui-se o quão importante é o planejamento da agricultura irrigada, pois a otimização do uso da água e a busca de melhor rentabilidade na agricultura, estimulam a integração de tecnologias de irrigação com sistemas de alta eficiência, reduzindo custos e maximizando a produção (PAZ et al.,2000).

Classificam-se os métodos de irrigação conforme a aplicação da água, estes métodos são: irrigação por superfície, irrigação subterrânea, irrigação por aspersão e irrigação localizada (MANTOVANI; BERNARDO; PALARETTI, 2009). Sendo os principais sistemas de irrigação localizada as irrigações por microaspersão e por gotejamento, onde a diferença entre esses dois emissores de marcas iguais ou diferentes podem causar variações significativas na vazão do sistema (SANTIAGO et al., 2012). 
Segundo Silva (2019), a prática da irrigação localizada comparada aos demais, sistemas de irrigação, vem crescendo destacadamente no cenário mundial mostrando-se um mercado promissor e tem particularmente na região semiárida maior aplicação devido ao seu uso proporcionar uniformidade e eficiência, além de minimizar os riscos de salinização.

$\mathrm{Na}$ irrigação localizada os emissores são conceituados como pequenos dispositivos que tem como função distribuir a água, estes são projetados para dissipar a pressão e descarregar constantemente um fluxo de água pequeno e uniforme. A microaspersão é um sistema intermediário entre a aspersão convencional e o gotejamento e foi idealizada orginalmente para substituir o gotejamento em áreas com solos arenosos, onde se necessita de bulbos molhados.

Clark et al. (2007), cita que o desempenho dos sistemas de irrigação implica em um adequado projeto hidráulico. Segundo Keller e Bliesner (1990), para dimensionar de forma correta o sistema faz-se necessário conhecer as características hidráulicas dos emissores, pois o manejo inadequado da irrigação resultará prejuízos referentes a gastos excessivos com água, e com isso, é provável que ocorra a lixiviação de nutrientes e até uma possível salinização do solo (VILAS BOAS et al., 2011b). Portanto, o uso correto da irrigação se torna indispensável, uma vez que este pode ser ajustado às condições de cada cultura. Souza et al.(2018) corrobora com Vilas Boas et al. (2011a) onde ambos destacam vantagens no sistema de irrigação por microaspersão, onde este possui uma alta eficiência e requerem baixa pressão para funcionamento além de apresentarem facilidade de operação e bom controle da umidade e da aeração do solo além da possibilidade de ser usada em qualquer tipo de solo; proporcionar um maior rendimento e qualidade no uso da água (assim como citada anteriormente); e promover uma maior eficiência no manejo da adubação (fertirrigação) (AYARS, 2006).

Um fator de grande relevância é a uniformidade de distribuição de água, esta informação é importante para a avaliação de sistemas de irrigação localizada, tanto no dimensionamento quanto na fase de operação do sistema (SANTOS et al., 2013). A uniformidade de um sistema de irrigação é uma medida quantitativa da vazão dos emissores ao longo de uma linha lateral ou de uma subunidade de irrigação, sendo o dimensionamento da linha lateral extremamente importante, pois a mesma tem a função de levar a água até os emissores que distribuem a água para as plantas (JAIN; SINGH; SINGH, 2002). A uniformidade de distribuição é um dos principais fatores de influência na produtividade agrícola da área irrigada. A uniformidade é influenciada pelas diferenças de pressão, desgaste 
do sistema e entupimento, principalmente quando utilizados fertilizantes. Fatores como o espaçamento entre os emissores/aspersores e fatores externos, como o clima e a topografia, além das variações de fabricação também afetam a uniformidade (ANDRADE et al., 2017).

\section{Metodologia}

O projeto foi dimensionado a partir de simulações matemáticas para um emissor tipo microaspersor vertical da marca Senninger, bocais Rosa e Gelo (Tabela 1), pelo qual foi avaliado nos mesmo o comportamento hidráulico do gradiente de pressão e da variação da vazão ao longo da linha lateral.

Tabela 1: Pontos da equação vazão-pressão dos microaspersores verticais Senninger.

Fonte: Própria (2019)

\begin{tabular}{cccc}
\hline \multicolumn{2}{c}{ Bocal Rosa } & \multicolumn{2}{c}{ Bocal Gelo } \\
\hline $\begin{array}{c}\text { H } \\
\text { (m.c.a) }\end{array}$ & $\begin{array}{c}\mathbf{q} \\
(\mathbf{L} / \mathbf{h})\end{array}$ & $\begin{array}{c}\mathbf{H} \\
\text { (m.c.a) }\end{array}$ & $\begin{array}{c}\mathbf{q} \\
(\mathbf{L} / \mathbf{h})\end{array}$ \\
\hline 10 & 22,88 & 10 & 54,53 \\
15 & 28,58 & 15 & 66,19 \\
20 & 33,46 & 20 & 75,94 \\
25 & 37,82 & 25 & 84,49 \\
30 & 41,8 & 30 & 92,18 \\
\hline
\end{tabular}

A curva vazão-pressão do emissor selecionado é regida pela Equação 1, proposta por Keller \& Karmeli (1974), que afirmaram a dependência que a vazão tem da pressão de operação, em que, k e x são coeficientes de ajuste. Ademais, quanto menor o valor do coeficiente $\mathrm{x}$, menor é a influência da pressão na vazão do sistema e assim em situação ideal $\mathrm{x}=0$.

$$
q=k \times h^{x}
$$

Em que,

$\mathrm{q}=$ Vazão do emissor $(\mathrm{l} / \mathrm{h})$;

$\mathrm{h}=$ Pressão de serviço do emissor (m.c.a);

$\mathrm{k}=$ constante de proporcionalidade que caracteriza cada emissor (adimensional);

$\mathrm{x}=$ Expoente, determinado pelo regime de escoamento (adimensional).

Os valores dos coeficientes da equação supracitada foram determinados utilizando os dados fornecidos pelo fabricante, também anteriormente citados (Tabela 2).

Tabela 2: Constante de proporcionalidade e expoente da Equação 1 para os microaspersores de bocal rosa e gelo.

\begin{tabular}{crrr}
\multicolumn{2}{c}{ Bocal Rosa } & & \multicolumn{2}{c}{ Bocal Gelo } \\
\hline $\mathbf{x}$ & $\mathbf{K}$ & $\mathbf{x}$ & $\mathbf{k}$
\end{tabular}


Fonte: Própria (2019)

\begin{tabular}{llll}
0,5485 & 6,467 & 0,4779 & 18,14 \\
\hline
\end{tabular}

As Tabelas 3 e 4 apresentam os dados de entrada para o desenvolvimento dos projetos em relação aos microaspersores de bocais rosa e gelo da Senninger, onde a partir dos mesmos foram feitas as simulações hidráulicas para o dimensionamento da linha lateral. Para poder simular o perfil de pressão ao longo da linha lateral, adotou-se o modelo de dimensionamento proposto por Wu e Gitlin (1975). De acordo com o modelo, os demais dados necessários para o dimensionamento do projeto foram calculados a partir do software Microsoft Excel 2010.

Tabela 3: Dados de entrada e cálculos preliminares para o dimensionamento da linha lateral do emissor de bocal Rosa.

\begin{tabular}{ccc} 
Dados de entrada & & \\
\hline Tamanho da Linha (L): & 59 & $\mathrm{~m}$ \\
\hline Número de Microaspersores (N): & 18 & \\
Espaçamento entre Microaspersores (Se): & 3,30 & $\mathrm{~m}$ \\
Diâmetro da Linha Lateral (d): & 13,00 & $\mathrm{~mm}$ \\
Vazão do emissor (q): & 33 & $1 / \mathrm{h}$ \\
Vazão da Linha Lateral (Q): & 595,39 & $1 / \mathrm{h}$ \\
Temperatura (T): & 20 & $\mathrm{~m} . \mathrm{c} . \mathrm{a}$ \\
Pressão de Serviço no inicio da Linha Lateral $(\mathrm{H} 0):$ & 25,00 & ${ }^{\circ} \mathrm{C}$ \\
Velocidade de escoamento (v): & 1,25 & $\mathrm{~m} / \mathrm{s}$ \\
Área da Tubulação (A): & 0,00013273 & $\mathrm{~m}{ }^{2}$ \\
Viscosidade Cinemática da água $(\gamma):$ & 0,00000090 & $\mathrm{~m}^{2} / \mathrm{s}$ \\
Coeficiente (m) & 1,75 & \\
Variação máxima de pressão permitida $(\Delta \mathrm{H})$ & 1,1898 & $\%$ \\
\hline
\end{tabular}

Tabela 4: Dados de entrada e cálculos preliminares para o dimensionamento da linha lateral do emissor de bocal Gelo.

\begin{tabular}{|c|c|c|}
\hline \multicolumn{3}{|l|}{ Dados de entrada: } \\
\hline Tamanho da Linha (L): & 36,65 & $\mathrm{~m}$ \\
\hline Número de Microaspersores $(\mathrm{N})$ : & 11,10 & unid. \\
\hline Espaçamento entre Microaspersores (Se): & 3,30 & $\mathrm{~m}$ \\
\hline Diâmetro da Linha Lateral (d): & 13,00 & $\mathrm{~mm}$ \\
\hline Vazão do emissor (q): & 75,94 & $1 / \mathrm{h}$ \\
\hline Vazão da Linha Lateral (Q): & 843,30 & $1 / \mathrm{h}$ \\
\hline Pressão de Serviço no inicio da Linha Lateral (H0): & 20,00 & m.c.a \\
\hline Temperatura $(\mathrm{T})$ : & 25,00 & ${ }^{\circ} \mathrm{C}$ \\
\hline Velocidade de escoamento (v): & 1,76 & $\mathrm{~m} / \mathrm{s}$ \\
\hline Área da Tubulação (A): & 0,00013273 & $\mathrm{~m}^{2}$ \\
\hline Viscosidade Cinemática da água $(\gamma)$ : & 0,00000090 & $\mathrm{~m}^{2} / \mathrm{s}$ \\
\hline Coeficiente (m) & 1,75 & \\
\hline
\end{tabular}


Variação máxima de pressão permitida $(\Delta \mathrm{H})$ $1,2207 \%$

As perdas de carga e pressões dos sistemas correspondentes ao bocal rosa e gelo, conforme Wu \& Gitlin (1975), estão dispostas nas tabelas 5 e 6.

Tabela 5: Cálculos das perdas de carga e das pressões da linha lateral com microaspersor de bocal Rosa

\begin{tabular}{ccc} 
Diâmetro comercial da tubulação $(\mathbf{D c})$ & $\mathbf{1 3 , 0 0}$ & $\mathbf{m m}$ \\
\hline hf com novo diâmetro & 9,76 & $\mathrm{~m} . \mathrm{c} . \mathrm{a}$ \\
hf '(L) real & 3,55 & $\mathrm{~m} . \mathrm{c} . \mathrm{a}$ \\
hf médio & 2,60 & $\mathrm{~m} . \mathrm{c} . \mathrm{a}$ \\
Ponto de Ocorrência da pressão média: & 22,37 & $\mathrm{~m}$ \\
Pressão no início da linha lateral (Ho) & 22,60 & $\mathrm{~m} . \mathrm{c} . \mathrm{a}$ \\
Pressão média: & 7,12 & m.c.a \\
Altura Manométrica Total $(\mathrm{H}):$ & 27,12 & m.c.a \\
\hline
\end{tabular}

Tabela 6: Cálculos das perdas de carga e das pressões da linha lateral com microaspersor de bocal Gelo.

\begin{tabular}{ccc} 
Diâmetro comercial da tubulação (Dc) & $\mathbf{1 3 , 0 0}$ & $\mathbf{m m}$ \\
(DI) \\
\hline hf & 11,20 & m.c.a \\
hf'(L) real & 4,07 & m.c.a \\
hf médio & 2,99 & m.c.a \\
Ponto de Ocorrência da pressão média: & 13,96 & $\mathrm{~m}$ \\
Pressão no início da linha lateral (Ho) & 22,99 & m.c.a \\
Pressão média: & 8,18 & m.c.a \\
Altura Manométrica Total $(\mathrm{H}):$ & 28,18 & m.c.a \\
\hline
\end{tabular}

\section{Resultados e Discussão}

A curva característica do microaspersor de bocal Rosa está apresentada na Figura 1, cujo ajuste resultou na equação $\mathrm{y}=6,47 \mathrm{X}^{0,5485}$, com coeficiente de determinação $\left(\mathrm{R}^{2}\right)$ de 1 . No intervalo de $0,5<\mathrm{x}<1,0$ o regime de escoamento varia de instável a laminar, verificandose maior influência das variações de pressão sobre a vazão (KELLER \& KARMELLI, 1974). 
Figura 1: Curva e equação característica vazão versus pressão do microaspersor bocal Rosa.

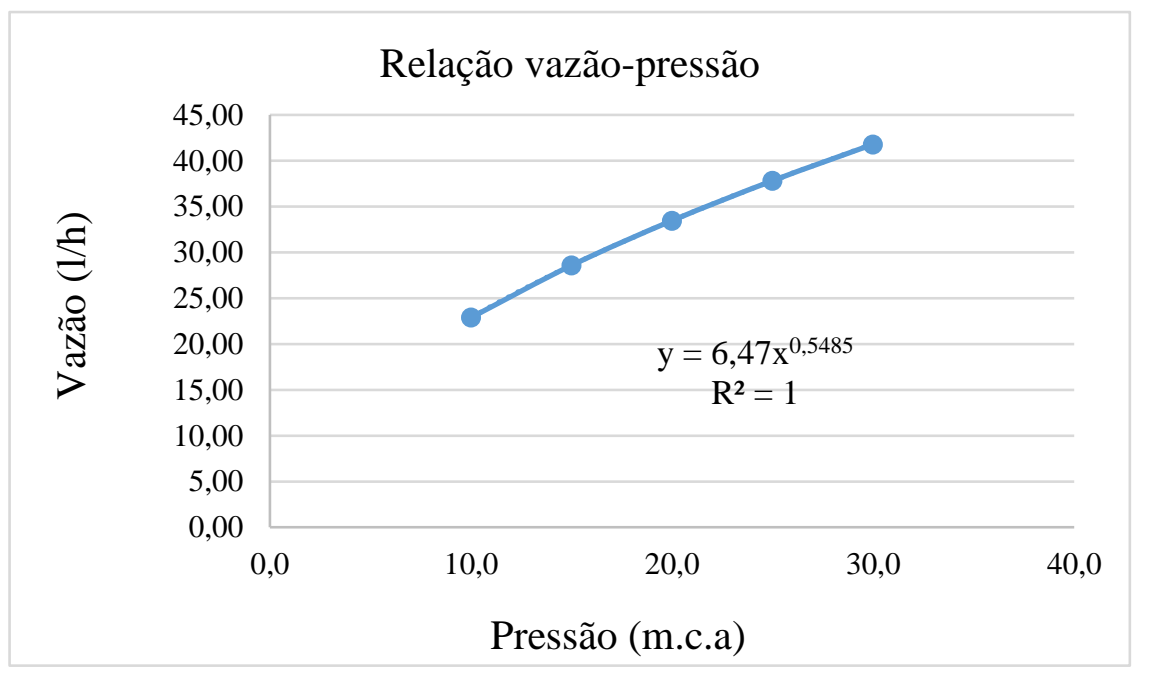

Fonte: Própria (2019)

O microaspersor de bocal gelo, por sua vez, apresenta curva vazão-pressão (Figura 2), conforme a equação $y=18,14 X^{0,4779}$. Para Keller \& Karmelli (1974), o expoente " $x$ " caracteriza o regime de fluxo e a relação vazão versus pressão do emissor, de modo que, no intervalo de $0<\mathrm{x}<0,5$ o regime de escoamento varia de turbulento a plenamente turbulento, e a vazão sofre menos influência da variação da pressão. Pizarro (1990) Destaca que um microaspersor perfeito teria o expoente $\mathrm{x}=0$ (autocompensante). Nesse sentido, o fluxo em questão pode ser caracterizado como um regime de escoamento que varia de instável a laminar, verificando-se maior influência das variações de pressão sobre a vazão.

Figura 2 - Curva e equação característica vazão versus pressão do microaspersor bocal Gelo.

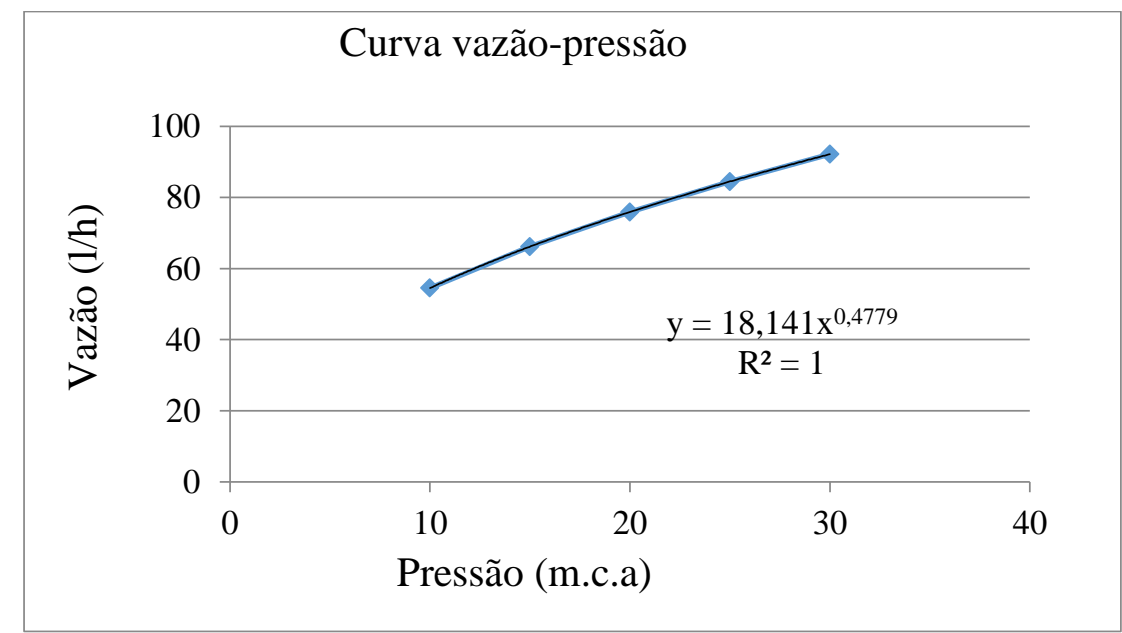

Fonte: Própria (2019) 
Aplicou-se a simulação para o bocal Rosa uma variação no gradiente de pressão $(\mathrm{H})$ entre 10,0 a 30,0 m.c.a. As vazões correspondentes (q), apresentaram tendência crescente do valor da pressão, variando de 22,88 a 41,80 L.h ${ }^{-1}$. Observando o resultado da modelagem e a variação dos dados, é possível confirmar a relação diretamente proporcional entre a pressão exercida pela bomba e a vazão do sistema de irrigação.

A Figura 3 representa o comportamento hidráulico da vazão e da pressão em cada ponto da linha lateral, para o microaspersor de bocal Rosa e Figura 4 para o microaspersor de bocal Gelo. É possível perceber que a relação vazão versus pressão decresceu ao longo do comprimento da linha. A quantidade de água aplicada e a uniformidade de distribuição são informações importantes para o dimensionamento ideal de qualquer sistema de irrigação localizada.

Figura 3 - Gradiente de energia (pressão) na linha lateral na posição dos microaspersores de bocal Rosa.

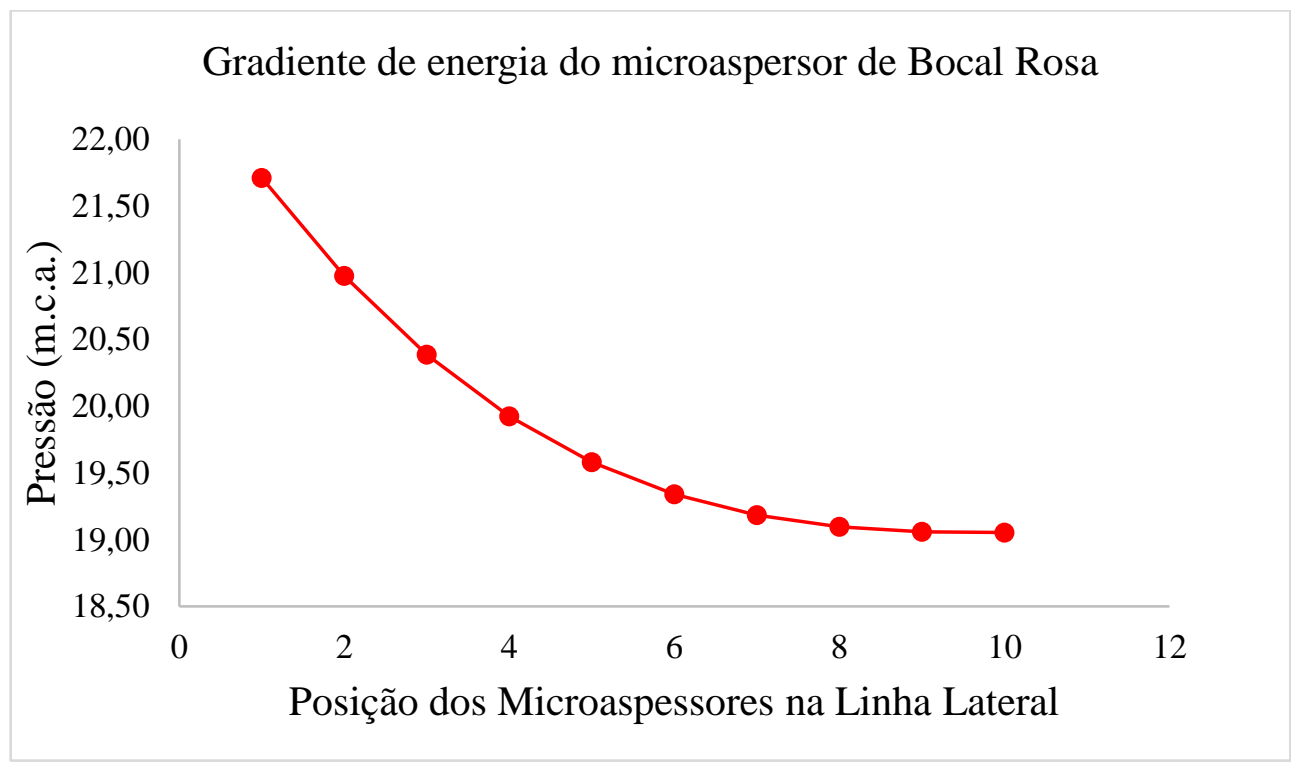

Fonte: Própria (2019) 
Figura 4 - Variação de vazão na linha lateral na posição dos microaspersores de bocal Rosa.

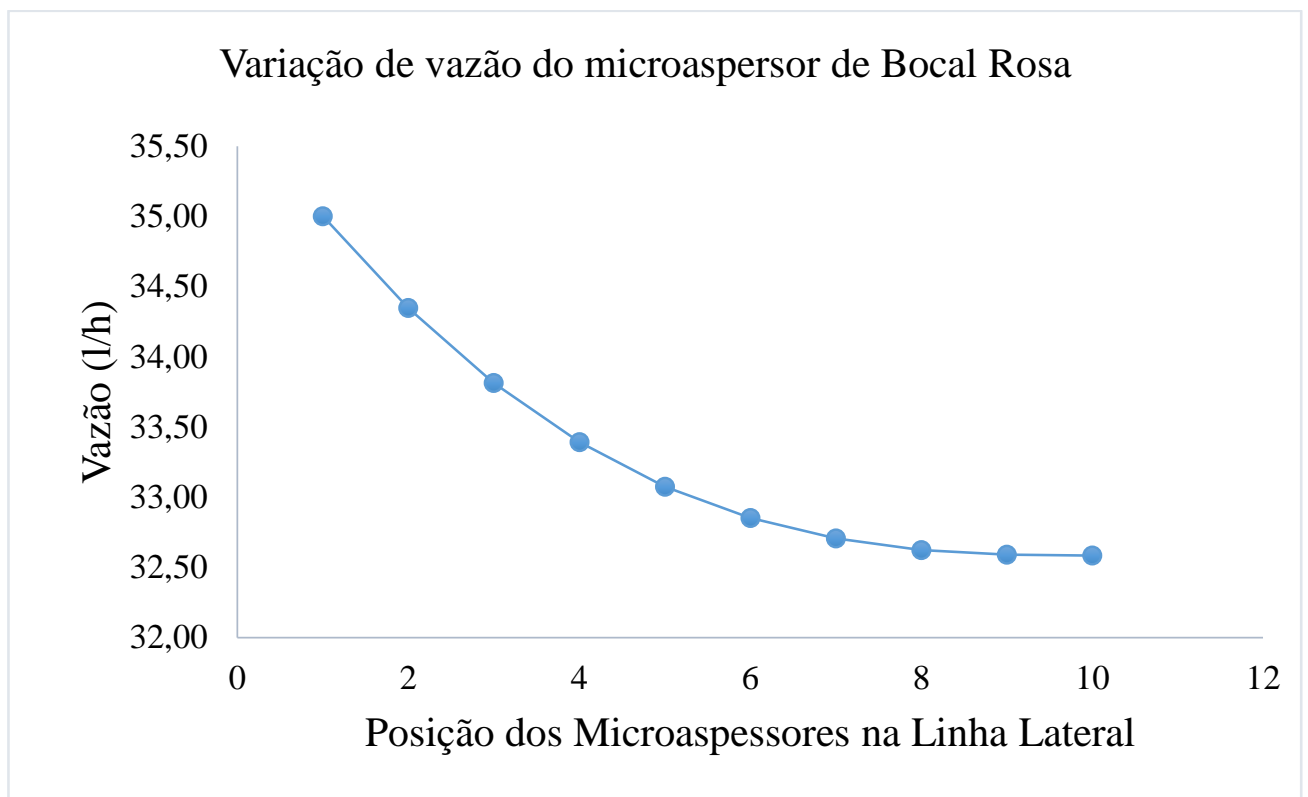

Fonte: Própria (2019)

As figuras 5 e 6 apresentam a relação da vazão-pressão em função dos emissores distribuídos na linha lateral, de acordo com a simulação matemática obtida anteriormente. Assim, pode-se observar o comportamento hidráulico da vazão e da pressão em função dos emissores, onde a relação vazão-pressão decresceu ao longo da linha lateral. A quantidade de água aplicada e a uniformidade de distribuição pelos emissores são informações importantes para o dimensionamento e manejo racional de um sistema de irrigação localizada. Neste sistema, a uniformidade de aplicação de água ao longo da linha lateral está intimamente relacionada com a variação de pressão e vazão dos emissores, a qual é uma consequência das perdas de energia por atrito e pelas inserções dos emissores, do ganho ou perda de energia devido à topografia da superfície do solo, e da qualidade da matéria prima e dos processos de fabricação dos emissores (KELLER; KARMELI, 1974 apud ALMEIDA; BOTREL, 2008). 
Figura 5 - Gradiente de energia (pressão) na linha lateral na posição dos microaspersores de bocal Gelo.

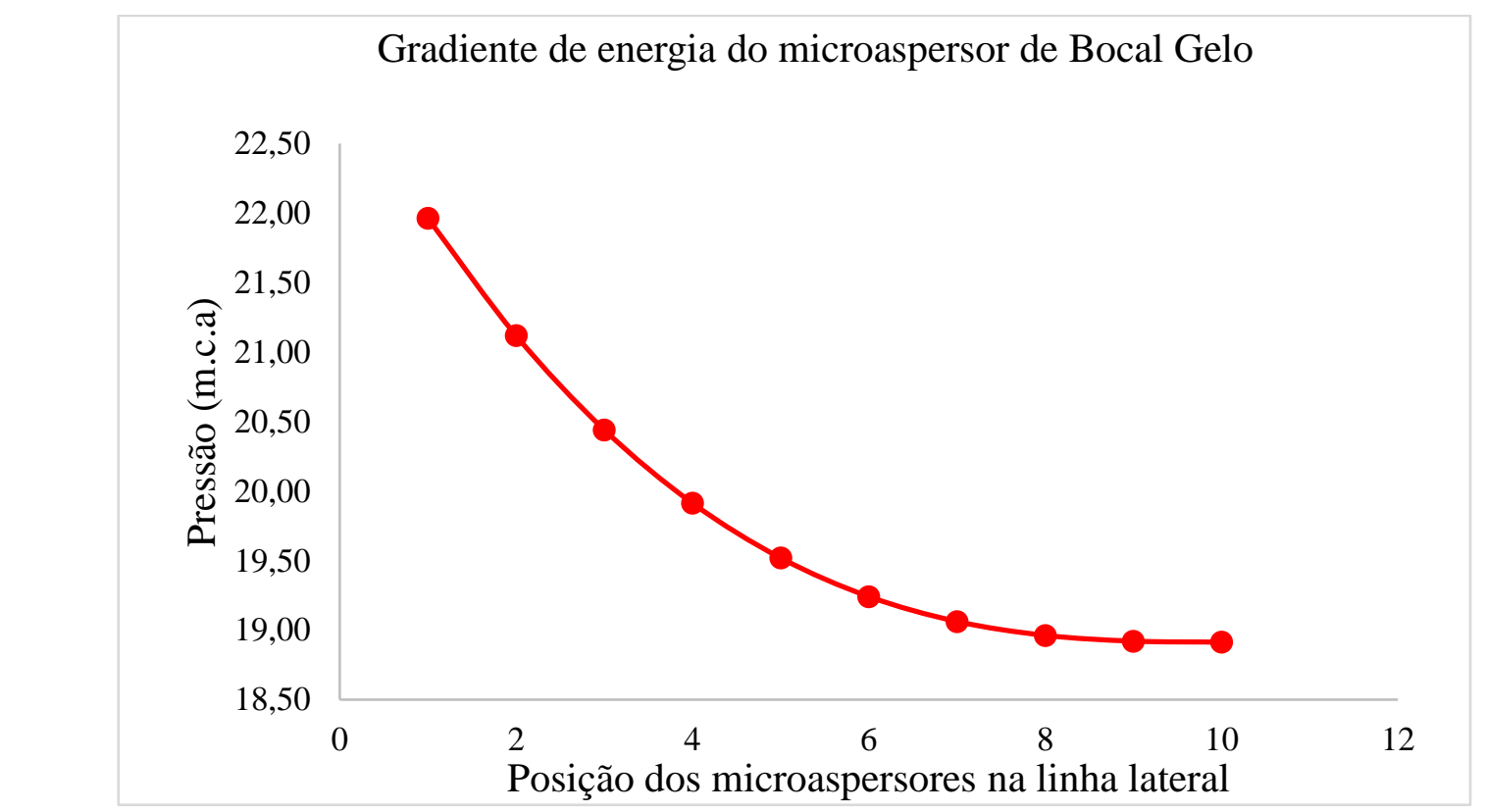

Fonte: Própria (2019)

Figura 6 - Gradiente de energia (pressão) na linha lateral na posição dos microaspersores de bocal Gelo.

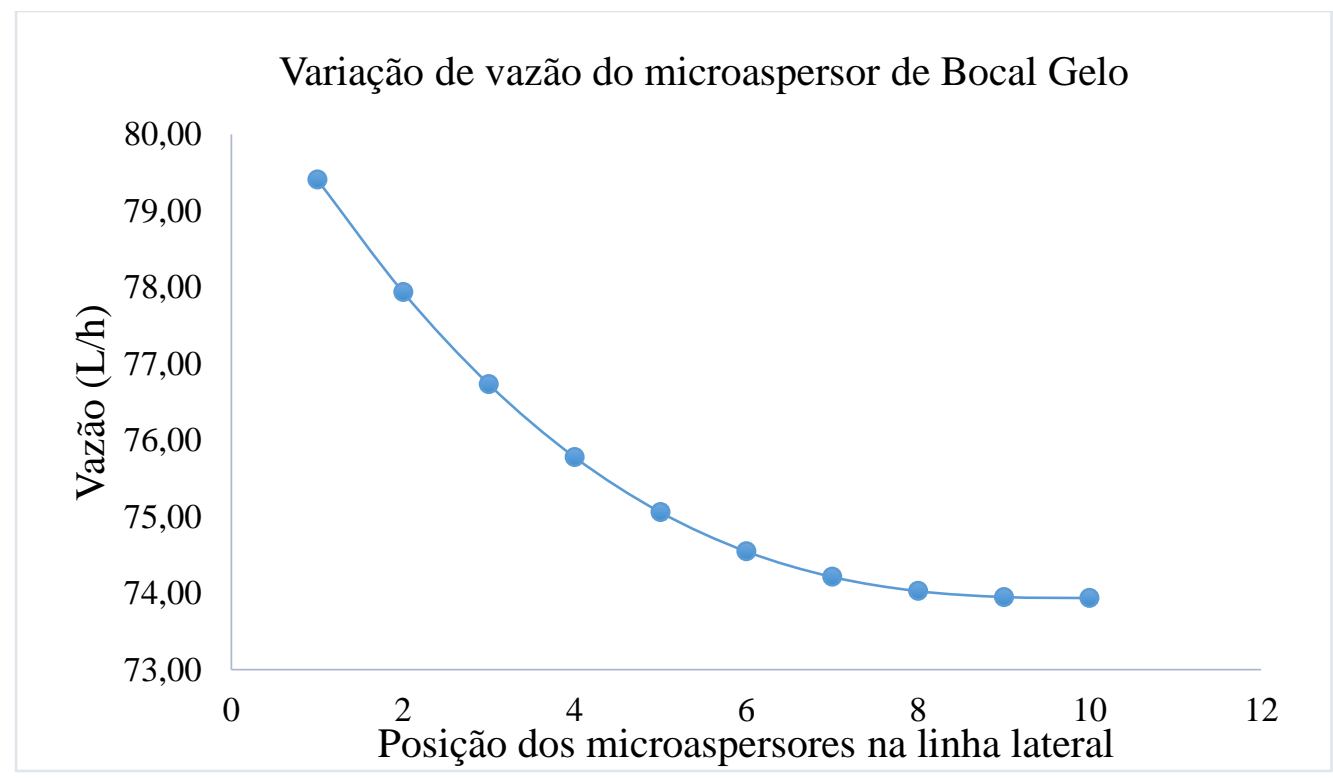

Fonte: Própria (2019)

Nesse tipo de sistema, a variação de pressão e vazão dos emissores normalmente é consequência das perdas de energia resultantes do atrito e inserções dos emissores, ganho ou perda de energia devido à topografia do terreno, e da qualidade da matéria prima e dos processos de fabricação dos emissores (KELLER; KARMELI, 1974, ALMEIDA; BOTREL 2010). 


\section{Conclusões}

A aplicação do modelo de Wu e Gitlin (1975) mostrou-se eficaz no dimensionamento do gradiente de pressão e vazão ao longo da linha lateral para os microaspersores de acordo com o comportamento hidráulico do sistema.

Os emissores apresentaram boas características hidráulicas a partir dos coeficientes de descargas (x). O microaspersor bocal Rosa apresentou uma variação de instável a laminar. O microaspersor bocal Gelo apresentou um regime turbulento a totalmente turbulento com a vazão sofrendo menos influência da variação da pressão. Este resultado pode estar relacionado com a distribuição da água ao longo da linha lateral.

\section{Referências}

ALMEIDA, C. D. G. C.; BOTREL, T. A. Determinação do diâmetro de microtubos em irrigação localizada. Revista Brasileira de Ciências Agrárias, v. 5, n. 3, p. 413-417, 2010.

ANA - AGÊNCIA NACIONAL DE ÁGUAS. Atlas Irrigação: Uso da Água na Agricultura Irrigada. Brasília, 2017. 86 p. Disponível em: <http://arquivos.ana.gov.br/imprensa/ publicacoes/AtlasIrrigacao-UsodaAguanaAgriculturaIrrigada.pdf>. Acesso em: 22 mai 2019.

ANDRADE, D. F.; OGLIARI, P. J. Estatística para as ciências agrárias e biológicas: Com noções de experimentação. 3 ed. rev. e ampl. Florianópolis: Editora da UFSC, 2013. 478 p.

ANDRADE, M. G.; VILAS BOAS, M. A.; SIQUEIRA, J. A. C.; SATO, M.; DIETER, J.; HERMES, E.; MERCANTE, E. Uniformity microsprinkler irrigation system using statistical quality control. Ciência Rural, v. 47, n. 4, p. 1-7, 2017.

AYARS, J. E.; BUCKS, D. A.; LAMM, F. R.; NAKAYAMA, F. S. Introduction. In: Freddie R. Lamm; James E. Ayars; Francis S. Nakayama. Microirrigation for crop production: Design, Operation, and Management. 1st ed. Amsterdam: Elsevier Science, 2006.

CASTIBLANCO, C. J. M. Modelo computacional para o dimensionamento da subunidade em irrigação localizada. 2013. Tese (Doutor em Ciências) - Escola Superior de Agricultura “Luiz de Queiroz”, Universidade de São Paulo, Piracicaba, 2013.

CHRISTIANSEN, J. E. Irrigation by sprinkling. California: University of California, 1942. $124 \mathrm{p}$.

CLARK, G. A.; HAMAN, D. Z.; PROCHASKA, J. F.; YITAYEW, M. General system design principles. In: Freddie R. Lamm; James E. Ayars; Francis S. Nakayama. Microirrigation for crop production: Design, Operation, and Management. 1st ed. Amsterdam: Elsevier Science, 2006. 
D‘ISEP, C. F. M. Água juridicamente sustentável. São Paulo: Editora Revista dos Tribunais, 2010. 317 p.

FAO - FOOD AND AGRICULTURE ORGANIZATION OF THE UNITED NATIONS. The state of the world's land and water resources for food and agriculture: Managing systems at risk. New York: Earthscan, 2011. 380 p. Disponível em: <http://www.fao.org/3/ai1688e.pdf>. Acesso: 20 maio 2019.

FGV - FUNDAÇÃO GETÚLIO VARGAS. Estudo sobre eficiência do uso da água no Brasil: análise do impacto da irrigação na agricultura brasileira e potencial de produção de alimentos face ao aquecimento global. Rio de Janeiro: GV Agro, 2016. 17 p. Disponível em: $<$ https://gvagro.fgv.br/pesquisa>. Acesso em: 21 mai 2019.

FRIZZONE, J. A.; FREITAS, P. S. L.; REZENDE, R.; FARIA, M. A. Microirrigação: Gotejamento e Microaspersão. Maringá: Eduem, 2012. 356 p.

HERRERO, M.; THORNTON, P. K.; NOTENBAERT, A. M.; WOOD, S.; MSANGI, S.; FREEMAN, H. A.; BOSSIO, D.; DIXON, J.; PETERS, J.; VAN DE STEEG, J.; LYNAM, J.; PARTHASARATHY RAO, P.; MACMILLAN, S.; GERARD, B.; MCDERMOTT, J.; SERÉ, C.; ROSEGRANT, M.. Smart investments in sustainable food production: revisiting mixed crop-livestock systems. Science, v. 327, n. 5967, p. 822-825, 2010.

JAIN, S. K.; SINGH, K. K.; SINGH, R. P. Microirrigation Lateral Design using Lateral Discharge Equation. Journal of Irrigation and Drainage Engineering, v. 128, n. 2, p. 125$128,2002$.

KELLER, J.; BLIESNER, R. D. Sprinkler and trickle irrigation. New York: Springer US, 1990. $652 \mathrm{p}$.

KELLER, J.; KARMELI, D. Trickle irrigation design parameters. Transactions of the American Society of Agricultural and Biological Engineers, v. 17, n. 4, p. 0678-0684, 1974.

MANTOVANI, E. C.; BERNARDO, S.; PALARETTI, L. F. Irrigação: Princípios e Métodos. 3 ed. Viçosa: Editora UFV, 2009. 355 p.

MAUPIN, M. A.; KENNY, J. F.; HUTSON, S. S.; LOVELACE, J. K.; BARBER, N. L.; LINDSEYK. S. Estimated use of water in the United States in 2010. Virginia: U.S. Geological Survey, 2014, 56 p.

OFFOR, U. H.; ALABI, S. B. An accurate and computationally efficient friction factor model. Advances in Chemical Engineering and Science, v.6, n. 3, p. 237-245, 2016.

PAZ, V. P. S.; TEODORO, R. E. F.; MENDONÇA, F. C. Recursos hídricos, agricultura irrigada e meio ambiente. Revista Brasileira de Engenharia Agrícola e Ambiental, Campina Grande, v. 4, n. 3, p. 465-473, 2000. 
PIMENTA, B. D.; ROBAINA, A. D.; PEITER, W. M.; KIRCHNER, J. H.; BEN, L. H. B. Performance of explicit approximations of the coefficient of head loss for pressurized conduits. Revista Brasileira de Engenharia Agrícola e Ambiental, Campina Grande, v. 22, n. 5, p. 301-307, 2018.

PIZARRO, F. Riegos localizados de alta frecuencia: Goteo, microaspersión, exudación. 2 ed. Madrid: Ediciones Mundi-Prensa, 1990. 461p.

SANTIAGO, F. dos S.; FREITAS, R. R. L.; BLACKBURN, R. M.; SILVA, N. C. G. da.; RIBEIRO, G.A.; NANES, M. B.; MONTENEGRO, A. A. A.; MONTENEGRO, S. M. G. L. Caracterização hidráulica de diferentes microaspersores para uso na agricultura familiar no Semiárido Nordestino. In: XXII Congresso Nacional de Irrigação e Drenagem, 2012, Cascavel. Anais do Congresso Nacional de Irrigação e Drenagem. Cascavel: Associação Brasileira de Irrigação e Drenagem, 2012.

SANTOS, C. S.; SILVA, P. F.; ALVES, E. S.; SANTOS, M. A. L. Avaliação da uniformidade de distribuição de um sistema de irrigação por gotejamento. Revista Verde de Agroecologia e Desenvolvimento Sustentável, v. 8, n. 3, p. 10-16, 2013.

SILVA, M. V.; DIAS, I. C. G. M; ALMEIDA, G. L. P; BATISTA, P. H. D.; SILVA, R. A. B. Modelagem do comportamento hidráulico de microaspersores ao longo da linha lateral, Revista de Engenharia e Tecnologia, v.11, n.1, 2019.

SOUSA, J. S. C.; DANTAS NETO, J.; Equação explícita para cálculo do fator de atrito de Darcy-Weisbach em projetos de irrigação pressurizada. Revista Irriga, v. 19, n. 1, p. 137$148,2014$.

TAIZ, L.; ZEIGER, E.; MOLLER, I. M.; MURPHY, A. Fisiologia e Desenvolvimento Vegetal. 6. ed. Porto Alegre: Artmed, 2017. 888 p.

VILAS BOAS, R. C.; PEREIRA, G. M.; SOUZA, R. J. de.; CONSONI, R. Desempenho de cultivares de cebola em função do manejo da irrigação por gotejamento. Revista Brasileira de Engenharia Agrícola e Ambiental, v. 15, n. 2, p. 113-124, 2011 b.

VÖRÖSMARTY, C. J.; MCINTYRE, P.; GESSNER, M. O.; DUDGEON, D.; PRUSEVICH, A.; GREEN, P.; GLIDDEN, S.; BUNN, S. E.; SULLIVAN, C. A.; LIERMANN, C. R.; DAVIS, P. M. Global threats to human water security and river biodiversity. Nature. v. 467, p. 555-561, 2010.

WU, I. P. An assessment of hydraulic design of micro-irrigation systems. Agric. Agricultural Water Management, v. 32, N. 3, p. 275 -284, 1997.

WU, I. P.; GITLIN, H. M. Energy gradient line for drip irrigation laterals. Journal of the Irrigation and Drainage Division, v. 101, n. 4, p. 323-326, 1975.

WWDR - World Water Development Report. Fatos e dados do relatório mundial das Nações Unidas sobre o desenvolvimento dos recursos hídricos 4: o manejo dos recursos hídricos em condições de incerteza e risco. Brasília: Unesco, 2012. 17 p. Disponível em: 
<https://unesdoc.unesco.org/ark:/48223/pf0000215492_por?posInSet=1\&queryId=16ff18ed9c1c-42c1-9163-1ebc8d17b6b4>. Acesso: 21 mai 2019.

WWDR - World Water Development Report. Relatório mundial das Nações Unidas sobre desenvolvimento dos recursos hídricos 2018: soluções baseadas na natureza para a gestão da água, fatos e dados. Brasília: Unesco, 2018. 12 p. Disponível em: <http://unesdoc.unesco.org/images/0026/002615/261579por.pdf>. Acesso: 21 mai 2019.

YU, Y.; HUBACEK, K.; FENG, K.; GUAN, D. Assessing regional and global water footprints for the UK. Ecological Economics, v. 69, n. 5, p. 1140-1147, 2010.

ZANELLA, M. E. Considerações sobre o clima e os recursos hídricos do semiárido nordestino. Caderno Prudentino de Geografia, n. 36, volume especial, p. 126-142, 2014. 